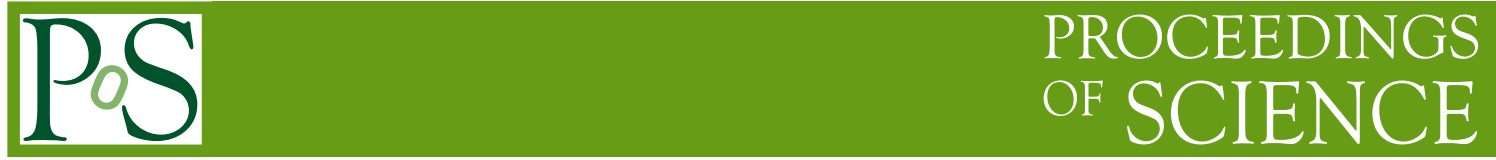

\title{
Charmonium Charged States
}

\author{
Marina Nielsen* ${ }^{\dagger}$ \\ Instituto de Física, Universidade de São Paulo, C.P. 66318, 05389-970 São Paulo, SP, Brazil \\ E-mail: mnielsen@if.usp.br
}

Some of the new charged chamonium states are studied using QCD sum rules. It is found that a tetraquark interpretation can be used to explain the masses and decay widths of these states.

XV International Conference on Hadron Spectroscopy-Hadron 2013

4-8 November 2013

Nara, Japan

\footnotetext{
* Speaker.

${ }^{\dagger}$ This work has been partly supported by FAPESP and CNPq-Brazil.
} 


\section{Introduction}

The year of 2013 might be remembered as the year that charmonium charged states were finally confirmed. The $Z_{c}^{+}(3900)$ was first observed by the BESIII collaboration in the $M\left(\pi^{ \pm} J / \psi\right)$ mass spectrum of the $Y(4260) \rightarrow J / \psi \pi^{+} \pi^{-}$decay channel [1]. This structure, was also observed at the same time by BELLE [2] and was confirmed by the authors of ref. [3] using CLEO-c data.

Up to now there are some experimental evidence for seven charged states, although not all of them may be independent or even exist. The first reported charmonium charged state, the $Z^{+}$(4430), was observed by Belle Collaboration in 2005, produced in $B^{+} \rightarrow K \psi^{\prime} \pi^{+}$[4]. Babar Collaboration [5] also searched the $Z^{-}$(4430) signature in four decay modes: $B \rightarrow \psi \pi^{-} K$, where $\psi=J / \psi$ or $\psi^{\prime}$ and $K=K_{S}^{0}$ or $K^{+}$. They concluded that there is no significant evidence for a signal peak in any of these processes. However, very recently the Belle Collaboration has confirmed the $Z^{+}$(4430) observation and has determined the preferred assignment of the quantum numbers to be $J^{P}=1^{+}[6]$. There are no reports of a $Z^{+}$signal in the $J / \psi \pi^{+}$decay channel.

Following the $Z^{+}$(4430) observation the Belle Collaboration reported the observation of two resonance-like structures, called $Z_{1}^{+}(4050)$ and $Z_{2}^{+}(4250)$, in the exclusive process $\bar{B}^{0} \rightarrow K^{-} \pi^{+} \chi_{c 1}$, in the $\pi^{+} \chi_{c 1}$ mass distribution [7]. Also in this case the BaBar colaboration has not confirmed these observations [8].

After these non confirmations, it was with great excitement that the hadron community heard about the observation of the $Z_{c}^{+}$(3900), mentioned above, that has been confirmed by three collaborations. Soon after the $Z_{c}^{+}(3900)$ observation, the BESIII related the observation of other three charges states:

- the $Z_{c}^{+}(4025)$, observed in the process $e^{+} e^{-} \rightarrow\left(D^{*} \bar{D}^{*}\right)^{ \pm} \pi^{\mp}$, at center of mass energy of 4.26 $\mathrm{GeV}[9]$,

- the $Z_{c}^{+}$(4020), observed in the process $e^{+} e^{-} \rightarrow \pi^{+} \pi^{-} h_{c}$, at center of mass energies from $3.90 \mathrm{GeV}$ to $4.42 \mathrm{GeV}$, in the $\pi^{ \pm} h_{c}$ mass spectrum [10],

- the $Z_{c}^{+}(3885)$, observed in the process $e^{+} e^{-} \rightarrow\left(D \bar{D}^{*}\right)^{ \pm} \pi^{\mp}$, at center of mass energy of 4.26 $\mathrm{GeV}[11]$.

Up to now it is not clear if the states $Z_{c}^{+}(3900)-Z_{c}^{+}(3885)$ and the states $Z_{c}^{+}(4025)-Z_{c}^{+}(4020)$ are the same states seen in different decay channels, or if they are independent states. The only comment from the experimental side is that if the $Z_{c}^{+}(3900)$ and $Z_{c}^{+}(3885)$ are the same state, then the ratio

$$
\frac{\Gamma\left(Z_{c}(3885) \rightarrow D \bar{D}^{*}\right)}{\Gamma\left(Z_{c}(3900) \rightarrow \pi J / \psi\right)}=6.2 \pm 1.1 \pm 2.7
$$

is determined [11].

All of these charged states can not be $c \bar{c}$ states and they are natural candidates for molecular or tetraquark states. These exotic states are allowed by the strong interactions, both at the fundamental level and at the effective level, and their absence in the experimentally measured spectrum has always been a mystery. The theoretical tools to address these questions are lattice QCD, chiral perturbation theory, QCD sum rules (QCDSR) and quark models. Here we discuss some of these new states using the QCDSR approach. 
The idea of the QCDSR formalism is to approach the bound state problem in QCD from short distances, where the dynamics is essentially perturbative, and move to larger distances including non-perturbative effects "step by step", using some approximate procedure to extract information on hadronic properties. It is based on identities between two and three-point Green functions, which connect hadronic observables with QCD fundamental parameters, such as quark masses, the strong coupling constant and quantities which characterize the QCD vacuum, the condensates.

In what follows we present some results of the QCDSR calculations on the new charmonium states, which we assume to be composed by four quarks either in a molecular or in a tetraquark configuration. For more details we refer the reader to ref. [12].

2. $Z_{c}^{+}(3900)$

The fisrt QCDSR calculation for the mass of the $X(3872)$ considered as a $I^{G}\left(J^{P C}\right)=0^{+}\left(1^{++}\right)$ tetraquark state was done in ref. [13], and a good agreement with the experimental mass was obtained. The $Z_{c}(3900)$ is interpreted here as the isospin 1 partner of the $X(3872)$. As in [14] we assume the quantum numbers for the neutral state in the isospin multiplet to be $I^{G}\left(J^{P C}\right)=1^{+}\left(1^{+-}\right)$. Therefore, the interpolating field for $Z_{c}^{+}(3900)$ is given by:

$$
j_{\alpha}=\frac{i \varepsilon_{a b c} \varepsilon_{d e c}}{\sqrt{2}}\left[\left(u_{a}^{T} C \gamma_{5} c_{b}\right)\left(\bar{d}_{d} \gamma_{\alpha} C \bar{c}_{e}^{T}\right)-\left(u_{a}^{T} C \gamma_{\alpha} c_{b}\right)\left(\bar{d}_{d} \gamma_{5} C \bar{c}_{e}^{T}\right)\right],
$$

where $a, b, c, \ldots$ are color indices, and $C$ is the charge conjugation matrix. Considering $S U(2)$ symmetry, the mass obtained in QCDSR for the $Z_{c}$ state is exactly the same one obtained for the $X(3872)$, as it happens in the case of $\rho$ and $\omega$ states. The mass obtained in [13] was $M=$ $(3.92 \pm 0.13) \mathrm{GeV}$, in a very good agreement with the $Z_{c}^{+}(3900)$ mass.

In order to study the $Z_{c}$ decay width we need to calculate the three-point function of the vertex of $Z_{c}^{+}$with the two other mesons in a given decay channel. As a result of the calculation we can obtain the coupling constant in the vertex which allows the evaluation of the decay width in each channel. In the framework of QCDSR this was done in ref. [15], where the $Z_{c}$ was assumed to be a tetraquark described by the current in Eq. (2.1). The authors of ref. [15] have considered four decay channels: $Z_{c}^{+}(3900) \rightarrow J / \psi \pi^{+}, Z_{c}^{+}(3900) \rightarrow \eta_{c} \rho^{+}, Z_{c}^{+}(3900) \rightarrow D^{+} \bar{D}^{* 0}$ and $Z_{c}^{+}(3900) \rightarrow \bar{D}^{0} D^{*+}$. The obtained couplings, with the respective decay widths, are given in Table I.

Table I: Coupling constants and decay widths in different channels.

\begin{tabular}{c|c|c}
\hline Vertex & coupling constant $(\mathrm{GeV})$ & decay width $(\mathrm{MeV})$ \\
\hline$Z_{c}^{+}(3900) J / \psi \pi^{+}$ & $3.89 \pm 0.56$ & $29.1 \pm 8.2$ \\
$Z_{c}^{+}(3900) \eta_{c} \rho^{+}$ & $4.85 \pm 0.81$ & $27.5 \pm 8.5$ \\
$Z_{c}^{+}(3900) D^{+} \bar{D}^{* 0}$ & $2.5 \pm 0.3$ & $3.2 \pm 0.7$ \\
$Z_{c}^{+}(3900) \bar{D}^{0} D^{*+}$ & $2.5 \pm 0.3$ & $3.2 \pm 0.7$ \\
\hline
\end{tabular}

Considering these four decay channels the obtained total width is $\Gamma=(63.0 \pm 18.1) \mathrm{GeV}$ for $Z_{c}(3900)$ which is in a good agreement with the two experimental values: $\Gamma=(46 \pm 22) \mathrm{MeV}$ from BESIII [1], and $\Gamma=(63 \pm 35) \mathrm{MeV}$ from BELLE [2]. From the results in Table I it is possible to evaluate the ratio

$$
\frac{\Gamma\left(Z_{c}(3900) \rightarrow D \bar{D}^{*}\right)}{\Gamma\left(Z_{c}(3900) \rightarrow \pi J / \psi\right)}=0.22 \pm 0.12
$$


which is completely different from the result in Eq. (1.1). Therefore, from a QCDSR calculation it is possible to conclude that, if the ratio in Eq. (1.1) is confirmed, the states $Z_{c}^{+}(3900)$ and $Z_{c}^{+}(3885)$ are not the same states.

\section{3. $Z_{c}^{+}(4025)$}

The $Z_{c}^{+}(4025)$ was observed in the $\left(D^{*} \bar{D}^{*}\right)^{+}$decay channel with a mass very close to the $D^{*} D^{*}$ threshold. Therefore, it might be explained as a $\left(D^{*} \bar{D}^{*}\right)^{+}$molecular state. A system of two vector mesons can possess a total spin-parity $0^{+}, 1^{+}$or $2^{+}$when interacting in s-wave. The $0^{+}$assignment is ruled out for $Z_{c}(4025)$ by spin-parity conservation for the $e^{+} e^{-} \rightarrow\left(D^{*} \bar{D}^{*}\right)^{ \pm} \pi^{ \pm}$ process. A QCDSR study for the $Z_{c}^{+}(4025)$ considered as a $\left(D^{*} \bar{D}^{*}\right)^{+}$molecular state was done in ref. [16]. To unambiguously separate the different spin-parity configurations of the $D^{*} \bar{D}^{*}$ systems, the authors of ref. [16] have applyed the spin projectors developed in [17], for a general current written in terms of two vector mesons.

The interpolating current corresponding to the $\bar{D}^{* 0} D^{*+}$ molecular state is given by:

$$
j_{\mu \nu}(x)=\left[\bar{c}_{a}(x) \gamma_{\mu} u_{a}(x)\right]\left[\bar{d}_{b}(x) \gamma_{v} c_{b}(x)\right],
$$

where $a, b$ denote the color indices. The two-point correlation function constructed with the current in Eq. (3.1) is

$$
\Pi_{\mu v \alpha \beta}\left(q^{2}\right)=i \int d^{4} x e^{i q x}\left\langle 0\left|T\left[j_{\mu v}(x) j_{\alpha \beta}^{\dagger}(0)\right]\right| 0\right\rangle .
$$

The $1^{+}$and $2^{+}$components of the correlation function written in Eq. (3.2) can be obtained using the following projectors

$$
\mathscr{P}^{(1)}=\frac{1}{2}\left(\Delta^{\mu \alpha} \Delta^{v \beta}-\Delta^{\mu \beta} \Delta^{v \alpha}\right), \quad \mathscr{P}^{(2)}=\frac{1}{2}\left(\Delta^{\mu \alpha} \Delta^{v \beta}+\Delta^{\mu \beta} \Delta^{v \alpha}\right)-\frac{1}{3} \Delta^{\mu v} \Delta^{\alpha \beta},
$$

where $\Delta_{\mu \nu} \equiv-g_{\mu \nu}+q_{\mu} q_{v} / q^{2}$. With these projectors, the masses obtained in ref. [16] for the $J^{P}=1^{+}$and $2^{+}$states are:

$$
\begin{aligned}
& M^{S=1}=(3950 \pm 105) \mathrm{MeV}, \\
& M^{S=2}=(3946 \pm 104) \mathrm{MeV} .
\end{aligned}
$$

These results indicate that these two states have very similar masses. Within the error bars, one of these could correspond to the recently observed $Z_{c}(4025)$ [9], although they could also correspond to a state below the threshold, in agreement with predicitions from ref. [18]. Resonance generation in the $D^{*} \bar{D}^{*}$ system has been studied within effective field theories $[18,19]$. In ref. [19] the $D^{*} \bar{D}^{*}$ system has been investigated with a formalism based on heavy quark symmetry and an isospin 1 resonance with $J^{P}=1^{+}$and mass ranging between 3950-4017 MeV has been found as dynamically generated. On the other hand, in the case of isospin 1, in ref. [18] only a state with $J^{P}=2^{+}$and mass $3900-3965 \mathrm{MeV}$ has been found in the $D^{*} \bar{D}^{*}$ system. As can be seen from Eq. (3.4), the results in $1^{+}$and $2^{+}$configurations are both in good agreement with those found in refs. [18, 19]. In particular, it was shown in ref. [22] that both assignments, a resonance with $J^{P}=1^{+}$or a bound state with $J^{P}=2^{+}$, are compatible with the data from ref. [9]. Besides, it was shown in ref. [22] that the experimental data can also be explained with just a pure wave-D background. However, this last possibility can be excluded with a study of the modification experienced by the $D^{*} \bar{D}^{*}$ invariant mass spectrum when the center of mass energy is varied from $\sqrt{s}=4.26 \mathrm{GeV}$ to $4.6 \mathrm{GeV}$. 


\section{Final Comments}

As a final remark, it is very important to find experimentally observable quantities which are sensitive to the quark content of the resonances. In refs. [20, 21], Cho et al. have suggest that studying the production of these exotic states in relativistic heavy ion collisions offer a promising resolution to this problem as yields of exotic hadrons are expected to be strongly affected by their structures. However, it might be very difficult to observe the new charmonium states discussed above in relativistic heavy ions collisions at LHC. Therefore, it is very important to find out other experimentally observable quantities which could be sensitive to the quark content of the resonances.

\section{References}

[1] M. Ablikim et al. [BESIII Collaboration], Phys. Rev. Lett. 110, 252001 (2013) [arXiv:1303.5949].

[2] Z.Q. Liu et al. [BELLE Collaboration], Phys. Rev. Lett. 110, 252002 (2013) [arXiv:1304.0121].

[3] T. Xiao, S. Dobbs, A. Tomaradze and K.K. Seth, arXiv:1304.3036.

[4] K. Abe et al. [Belle Collaboration], Phys. Rev. Lett., 2008, 100: 142001.

[5] B. Aubert et al. [BaBar Collaboration], Phys. Rev. D, 2009, 79: 112001.

[6] K. Chilikin et al. [Belle Collaboration], Phys. Rev. D 88, 074026 (2013) [arXiv:1306.4894].

[7] R. Mizuk et al. [Belle Collaboration], Phys. Rev. D 78, 072004 (2008).

[8] J. P. Lees et al. [BaBar Collaboration], Phys. Rev. D 85, 052003 (2012) [arXiv:1111.5919].

[9] M. Ablikim et al. [BESIII Collaboration], arXiv:1308.2760.

[10] M. Ablikim et al. [BESIII Collaboration], arXiv:1309.1896.

[11] M. Ablikim et al. [BESIII Collaboration], arXiv:1310.1163.

[12] N. Brambilla et al., Eur. Phys. J. C 71, 1534 (2011); M. Nielsen, F.S. Navarra and S.H. Lee, Phys. Rept. 497, 41 (2010), and references therein.

[13] R.D. Matheus, S. Narison, M. Nielsen and J.-M. Richard, Phys. Rev. D 75, 014005 (2007).

[14] L. Maiani, F. Piccinini, A.D. Polosa and V. Riquer, Phys. Rev. D 71, 014028 (2005).

[15] J. M. Dias et al. , Phys. Rev. D 88, 016004 (2013) [arXiv:1304.6433].

[16] K. P. Khemchandani, A. Martinez Torres, M. Nielsen and F. S. Navarra, arXiv:1310.0862.

[17] A. Martínez Torres, K. P. Khemchandani, M. Nielsen, F. S. Navarra and E. Oset, arXiv:1307.1724.

[18] R. Molina and E. Oset, Phys. Rev. D 80, 114013 (2009) [arXiv:0907.3043].

[19] F. -K. Guo, C. Hidalgo-Duque, J. Nieves and M. P. Valderrama, arXiv:1303.6608.

[20] S. Cho et al. [ExHIC Collaboration], Phys. Rev. Lett. 106, 212001 (2011) [arXiv:1011.0852].

[21] S. Cho et al. [ExHIC Collaboration], Phys. Rev. C 84, 064910 (2011) [arXiv:1107.1302].

[22] A. Martinez Torres, K. P. Khemchandani, F. S. Navarra, M. Nielsen and E. Oset, arXiv:1310.1119. 